\title{
Minimum Time Regulation of DC-DC Converters in Damping Mode with an Optimal Adjusted Sliding Mode Controller
}

\author{
Mohammad Javad Jafarian $^{*}$ and Jalal Nazarzadeh ${ }^{\dagger}$ \\ ${ }^{* \dagger}$ Faculty of Engineering, Shahed University, Tehran, Iran
}

\begin{abstract}
In this paper, a new development in the time optimal control theory in sliding mode control systems for multi-quadrant buck converters with a variable load is presented. In general, the closed-loop time optimal control system is applied to multi-quadrant buck converters for output regulation, so that an optimal switching surface is obtained. Moreover, an adjusted optimal sliding mode controller is suggested which adjusts the controller parameters to give an optimal switching surface. In addition, a description of the transient response of the closed-loop system is proposed and used to damp any output or input disturbances in minimum time. Numerical simulations and experimental results are employed to demonstrate that the output regulation time and transient performances of $\mathrm{dc} / \mathrm{dc}$ converters using the proposed technique are improved effectively when compared to the classical sliding mode control method.
\end{abstract}

Key words: Adjusted Optimal Switching Surface, DC Converters, Sliding Mode Control, Time Optimal Control

\section{INTRODUCTION}

Nowadays, nonlinear control methods are widely used to improve the dynamic performance of dynamical systems. An effective nonlinear robust control method is the sliding mode control (SMC) technique proposed for variable structure systems [1]. It is a popular control approach for systems with uncertainties or unknown disturbances. This control system is stable and robust. In sliding mode control systems, an input control with a high switching frequency is employed as a function of the instantaneous state values to see to it that the system trajectory stays on a selected switching surface. Moreover, a suitable input control keeps the system trajectory near the sliding surface until it reaches the equilibrium point [2], [3]. The switching surface plays an important role in the design of sliding mode controllers. Fast dynamic response and good transient performance of systems can be created by an appropriate choice of the switching function.

In addition, tracking errors can be eliminated or reduced to satisfactory values for practical applications in sliding mode

Manuscript received Jun. 2, 2011; revised Jun. 17, 2012

Recommended for publication by Associate Editor Byung-Cho Choi.

${ }^{\dagger}$ Corresponding Author: nazarzadeh@shahed.ac.ir

Tel: +9821-51212041, Fax:+9821-5522-8817, Shahed University

*Faculty of Engineering, Shahed University, Tehran, Iran controllers [4]. In most literature, the switching surface is selected as a linear function of state variables for simplicity [5], [6]. Therefore, with low dynamic performance in systems like the elimination of steady state errors, the switching surface function is improved by adding single or double PI controllers [7]. However, a PI controller is a lag compensation and it reduces the bandwidth of a closed-loop system. Like SMC systems, conditional stability occurs when a system has saturation in the controller. On the other hand, a class of optimization problems is the minimization of the transition time from any initial state to a desired point that is called minimum time optimal control systems.

Pontryagen's minimum principle is an open loop technique for time optimal control systems. In this approach, a time optimal control with an input constraint has the bang-bang property, and the input control depends on the initial and final conditions [8]. For the independence form of any initial and final state conditions, a closed-loop time optimal control method with the bang-bang property is introduced by using an optimal switching surface [8]. However, since the elimination of the time variable for system trajectory is very complicated, an analytical solution for obtaining the switching surface function is not possible for damped sinusoidal oscillator systems. To solve this problem, approximated or numerical methods are applied [9]. 
In harmonic oscillator systems, the optimal switching surface can be obtained by a set of fixed radial circles [10]. These circles only depend on the initial states and the input control values. However, the design and implementation of the optimal switching surface in stable damped sinusoidal oscillator systems are very complicated. In this case, the optimal switching surface is a set of dissimilar ellipsoid functions that are dependent on the system parameters and time, as well as, the initial states and input control values.

Any dc/dc converter is characterized by an inherently damped sinusoidal oscillator with the bang-bang property [11]. Thus nonlinear controllers, such as sliding mode controllers, are appropriate for application to dc/dc converters. On the other hand, applying sliding mode control technique to multi-quadrant buck converters has some disadvantages such as: a non-constant switching frequency and the presence of a steady state regulation error [5], [12]. Nevertheless, pulse-width-modulation (PWM) [6], [13], and adaptive feed-forward and feedback hysteresis band control [11] were suggested to achieve a fixed switching frequency. A single or double integral sliding surface method was proposed to improve the steady state regulation error [7], [14], [15].

In buck converters, load changing leads to a transient over/ under voltage and current. In the SMC regime, the transient performances of buck converters are improved by appropriate selection of the switching surface, i.e., substituting second order [15] or approximation of the hyperbola [16] functions for the first order linear function. However, for large amplitude disturbances or a low capacitance and a high inductance, second order and approximation of the hyperbola functions do not exhibit suitable transient characteristics because the existing condition cannot be satisfied. To improve the transient performance of the output voltage and to obtain an optimal time response for full load rejection, the authors of [10] proposed the optimal switching surface. In general, and especially in load changing without output load rejection, this method does not have optimal dynamical performance.

In this paper, a closed-loop time optimal control system is employed for systems with stable damped mode conditions and extended in the general case. It is also extended to switching multi-quadrant buck converters. Furthermore, an optimal switching surface for dc/dc converters is introduced. Unlike the SMC system, the optimal controller in the closed-loop form can eliminate tracking errors in minimum time for any output variation by using the optimal switching surface. Optimal controller parameters are also adapted to adjust the optimal switching surface corresponding to any load variation. Finally, to compare the capability of the optimal SMC system, some numerical simulations and experimental results are presented. The results indicate that, the transient and steady state performances of the time optimal dc/dc converters are effectively improved in comparison with the classical SMC systems.

\section{SLIDING MODE CONTROL SYSTEMS FOR OUTPUT REGULATION}

The sliding mode control technique is an effective nonlinear control method which has high-performance in terms of external disturbances. This technique, which is based on the measurement of instantaneous state variables, is suitable for variable structure systems. In practice, the switches of a dc/dc converter are in the "on" or "off" modes. Thus the input control of this system is a piecewise constant function in a SMC system in which the system trajectory stays on a switching surface by duty cycle control. Consider a linear time invariant dynamic system as follows:

$$
\dot{\mathbf{x}}(t)=\mathbf{A} \mathbf{x}(t)+\mathbf{B u}(t)
$$

where $\mathbf{x}(\mathrm{t})$ and $\mathbf{u}(\mathrm{t})$ are two vectors which represent the system state and control input. In addition, $\mathbf{A}$ and $\mathbf{B}$ are the system and input matrices, respectively. The input control constraint is given as:

$$
\mathbf{u}_{N}<\mathbf{u}(t)<\mathbf{u}_{P}
$$

where $\mathbf{u}_{N}$ and $\mathbf{u}_{P}$ are lower and upper limits of the input control. For designing classical SMC controllers in second-order dynamic systems, a dependant function of the states variables is defined as [3]:

$$
S_{s m}\left(x_{1}, x_{2}\right)=\beta_{1}\left(x_{1}(t)-x_{1}\left(t_{f}\right)\right)+\beta_{2}\left(x_{2}(t)-x_{2}\left(t_{f}\right)\right)
$$

where $\beta_{1}$ and $\beta_{2}$ are the sliding coefficients of the controlled system and $x_{1}\left(t_{f}\right)$ and $x_{2}\left(t_{f}\right)$ are the final values of the state variables. The switching surface for the sliding mode control system is a linear function as $S_{s m}\left(x_{1}, x_{2}\right)=0$. When the input switching frequency tends to infinity, the system trajectory stays on the switching surface and the time constant of the closed-loop system is $\beta_{1} / \beta_{2}$ [3].

Existing, hitting and stability conditions have been satisfied for implement the SMC in systems (1) [3]. The existing condition determines the region of the phase plane where the system trajectory slides on the switching surface. For a small region near the switching surface, if the system trajectory directs toward the sliding surface (2) by itself, the existing condition will be satisfied. To establish this condition the following inequality should be held:

$$
\lim _{S_{s m} \rightarrow 0} S_{s m}\left(x_{1}, x_{2}\right) \dot{S}_{s m}\left(x_{1}, x_{2}\right)<0
$$

where $\dot{S}_{s m}\left(x_{1}, x_{2}\right)$ is the time derivation of the switching surface. The coefficients of the sliding surface are selected in a manner that satisfies (4). When inequality (4) holds in the whole region of the phase plane, it suffices for the system trajectories to reach the sliding surface. Whereas, the hitting condition is the sufficient condition to reach the system trajectory of the sliding surface. The system trajectory can reach the switching surface 
when one of the following two conditions is satisfied:

$$
\begin{aligned}
& \mathbf{x}_{P} \in S_{s m}>0 \Rightarrow \mathbf{x}_{N} \in S_{s m}<0 \\
& \mathbf{x}_{P} \in S_{s m}<0 \Rightarrow \mathbf{x}_{N} \in S_{s m}>0
\end{aligned}
$$

where $\mathbf{x}_{N}$ and $\mathbf{x}_{P}$ are the steady state values of the state variables with input controls of $\mathbf{u}_{N}$ and $\mathbf{u}_{P}$, respectively. Considering (4) and (5), the control law for infinite switching frequency in the SMC can be determined as:

$$
\mathbf{u}(t)= \begin{cases}\mathbf{u}_{P} & S_{s m} \leq 0 \\ \mathbf{u}_{N} & S_{s m}>0\end{cases}
$$

System stability can be guaranteed for a second-order system, if its trajectory in the sliding regime directs toward a stable operating point [1]. In higher-order dynamic systems, stability should be verified by non-linear stability theorems. Therefore, in a second-order system, the stability of the closed-loop system can be guaranteed by selecting proper values for $\beta_{1}$ and $\beta_{2}$ in (3). Although the switching surface (3) guarantees system stability and robustness, it may not result in suitable transient characteristics. Thus some of the high dynamic performances can be obtained if the switching surface is selected in the optimal nonlinear form $S_{s m}\left(x_{1}, x_{2}\right)$.

\section{Minimum TIME OPTIMAL CONTROL SYSTEMS}

A class of optimization problems is called the minimum-time optimal control which minimizes the transition time from any initial state to the target set. A systematic approach to solve the time optimal problem is using the minimum principle of Pontryagin. In the minimum time problem, the cost functional is considered as:

$$
J=\int_{0}^{t_{f}} d t
$$

The system and input matrices in (1) for damped sinusoidal oscillator systems are:

$$
\mathbf{A}=\left(\begin{array}{cc}
-\xi \omega_{n} & \omega_{d} \\
-\omega_{d} & -\xi \omega_{n}
\end{array}\right) \quad B=\left(\begin{array}{l}
0 \\
1
\end{array}\right)
$$

where $\xi$ and $\omega_{n}$ are the damping ratio and the un-damped natural frequencies, respectively. In addition, $\omega_{d}$ is the damped natural frequency which is equal to:

$$
\omega_{d}=\omega_{n} \sqrt{1-\xi^{2}}
$$

The optimal switching surface is applied to extract the optimal control law for the second order system in (1) and (8) with $\omega_{n}>0,0<\xi<1$ and the constrained input control (2). By using (1) and (7,8), Hamiltonian's function $H(\mathbf{x}(t), \mathbf{u}(t), \mathbf{p}(t))$ is obtained as:

$$
H(\mathbf{x}(t), \mathbf{u}(t), \mathbf{p}(t))=1+\mathbf{p}^{T}(t)(\mathbf{A x}(t)+\mathbf{B u}(t))
$$

where $\mathbf{p}(t)$ is a vector of the costate variables which can be obtained as:

$$
\dot{\mathbf{p}}(t)=-\frac{\partial}{\partial \mathbf{x}} H(\mathbf{x}(t), \mathbf{u}(t), \mathbf{p}(t))=-\mathbf{A}^{T} \mathbf{p}(t)
$$

By substituting (8) into (11), the following is obtained:

$$
\left(\begin{array}{c}
\dot{p}_{1}(t) \\
\dot{p}_{2}(t)
\end{array}\right)=\left(\begin{array}{cc}
\xi \omega_{n} & \omega_{d} \\
-\omega_{d} & \xi \omega_{n}
\end{array}\right)\left(\begin{array}{l}
p_{1}(t) \\
p_{2}(t)
\end{array}\right)
$$

The time optimal control of system (1) and (8) with constrained input control (2) is followed by the bang-bang property. Thus optimal control is a piecewise constant function of time [17]. In addition, the input control is achieved by minimization of the Hamiltonian (10) subject to (2). Thus by considering (2) and (10), the time optimal control is obtained by:

$$
u(t)=\left\{\begin{array}{cc}
u_{p} & p_{2}(t)<0 \\
u_{N} & p_{2}(t)>0 \\
\text { Singular } & p_{2}(t)=0
\end{array}\right.
$$

The input control $u(t)$ in (13) is dependent on the sign of the costate variable $p_{2}(t)$. Then, for $0<\xi<1$ and $\omega_{n}>0$ in (12), the switching number of the input control is not limited. The costate variable $p_{2}(t)$ can be obtained by solving (12) with the non-singular input control. In this case, the following is obtained:

$$
p_{2}(t)=e^{\xi \omega_{n} t}\left(k_{1} \cos \omega_{d} t+k_{2} \sin \omega_{d} t\right)
$$

where $k_{1}$ and $k_{2}$ are two constant coefficients. Fig. 1 shows $p_{2}(t)$ and $u(t)$ with $\xi=0.1$ and $\omega_{n}=1 \mathrm{rad} / \mathrm{sec}$ for typical initial conditions. These results show that the sign of $p_{2}(t)$ is constant for $\pi / \omega_{d}$ units of time. Thus the input control will be constant for this duration. Since the initial values of the states of the costate variables are unknown, the closed-loop optimal control system (1) and (8) based on costate dynamic (12) is not realizable. Moreover, by using the convolution integral, the state variables of the system (1) and (8) can be expressed as:

$$
\mathbf{x}(t)=\Phi(t) \mathbf{x}(0)+\int_{0}^{t} \Phi(v-t) \mathbf{B} u(v) d v
$$

where $\mathbf{x}(0)$ is the initial condition of the system. In addition, $\Phi(t)$ is the transition matrix of the system which can be obtained in the under-damped condition $0<\xi<1$ as:

$$
\Phi(t)=e^{\mathbf{A} t}=e^{-\zeta \omega_{n} t}\left(\begin{array}{cc}
\cos \omega_{d} t & \sin \omega_{d} t \\
-\sin \omega_{d} t & \cos \omega_{d} t
\end{array}\right)
$$

By substituting (8) and (16) into (15), the state variables of the system can be expressed as:

$$
\begin{aligned}
x_{1}(t)= & e^{-\xi \omega_{n} t}\left(x_{1}(0) \cos \omega_{d} t+x_{2}(0) \sin \omega_{d} t-\right. \\
& \left.-\frac{u(t)}{\omega_{n}} \sin \left(\omega_{d} t+\theta\right)\right)+\frac{u(t)}{\omega_{c}} \\
x_{2}(t)= & e^{-\xi \omega_{n} t}\left(x_{2}(0) \cos \omega_{d} t-x_{1}(0) \sin \omega_{d} t-\right. \\
& \left.-\frac{u(t)}{\omega_{n}} \cos \left(\omega_{d} t+\theta\right)\right)+\frac{\xi}{\omega_{n}} u(t)
\end{aligned}
$$




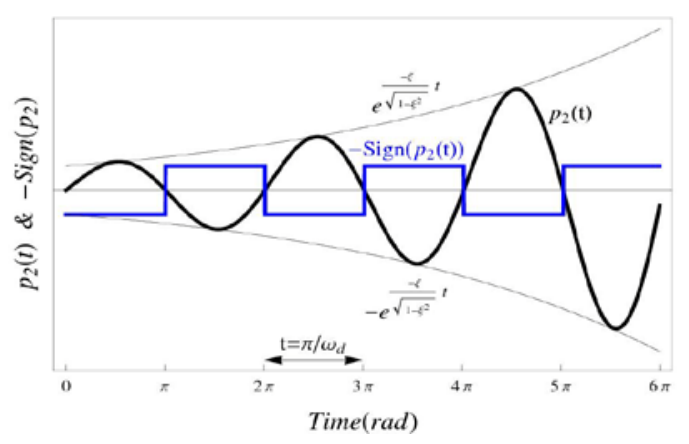

Fig. 1. $\quad p_{2}(t)$ and $u(t)$ for $\xi=0.1$ and $\omega_{n}=1 \mathrm{rad} / \mathrm{sec}$.

where:

$$
\theta=\tan ^{-1} \frac{\sqrt{1-\xi^{2}}}{\xi} \quad \omega_{c}=\frac{\omega_{n}}{\sqrt{1-\xi^{2}}}
$$

Since (17) is a nonlinear function of $t$, the system trajectory cannot be obtained by analytical elimination of the time variable in (17). The typical system trajectories in (17) are shown in Figs. 2-a and 2-b for the two input controls $u_{N}=0$ and $u_{P}=1$. The system trajectory is divided to different paths such as $C_{+}^{0}, \mathbb{C}_{+}^{1} C_{-}^{0}$ and $C_{-}^{1}$ which start from $\mathrm{A}$ at $t=0$ and is directed toward $\mathrm{B}$ at $t=\pi / \omega_{d}$ units of time, $\mathrm{C}$ at $t=2 \pi / \omega_{d}$ units of time and so on. Relation (14) shows that the optimal control should not be constant for more than $t=\pi / \omega_{d}$ units of time. Then, the two parts of the trajectory $C_{+}^{0}$ and $C_{-}^{0}$ are a set of state variables that can reach to the desired point in no more than $t=2 \pi / \omega_{d}$ units of time with the input constants $u(t)=u_{p}$ and $u(t)=u_{N}$, respectively. In the general case, similar to Fig. 2, the jth part of the system trajectories are called $C_{+}^{j}$ and $C_{-}^{j}$. Then the optimal switching surface for the time optimal control is constructed by $\lambda_{+}^{j}$ and $\lambda_{-}^{j}$. This optimal switching surface is obtained by the shifting and its inverse $C_{+}^{j}$ and $C_{-}^{j}$. A typical switching surface is shown in Fig. 2-b. Finally, the switching surface can be defined as:

$$
S_{\text {op }}\left(x_{1}, x_{2}\right)=\left\{\bigcup_{j=0}^{n} \lambda_{+}^{j}\right\} \cup\left\{\bigcup_{j=0}^{n} \lambda_{-}^{j}\right\}
$$

where $n$ is the number of system trajectory parts in the switching surface. When $n \neq 0$, any state variable on the $\lambda_{+}^{j}$ or $\lambda_{-}^{j}$ curve can be forced to $\lambda_{+}^{j-1}$ or the curve for $\lambda_{-}^{j-1}$ by the input control $u(t)=u_{p}$ and $u(t)=u_{N}$ in no more than $t=\pi / \omega_{d}$ units of time. Based on Fig. 2-b, the phase plane is divided into two parts, $S_{o p}\left(x_{1}, x_{2}\right)<0$ and $S_{o p}\left(x_{1}, x_{2}\right)>0$. Then the time optimal control $u^{*}(t)$ as a function of the state variables $\left(x_{1}, x_{2}\right)$ can be found as [17]:

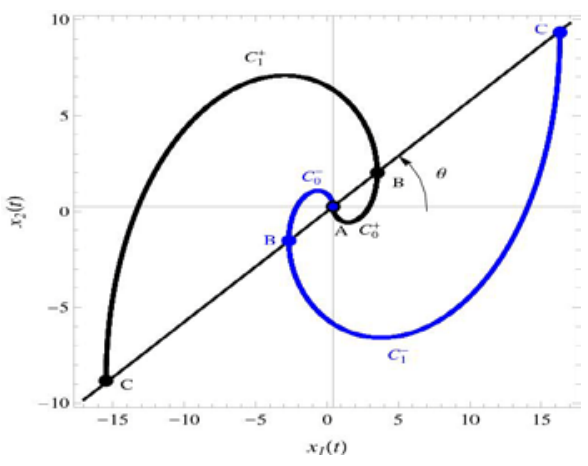

a) Trajectory with $u(t)=0$ and $u(t)=1$

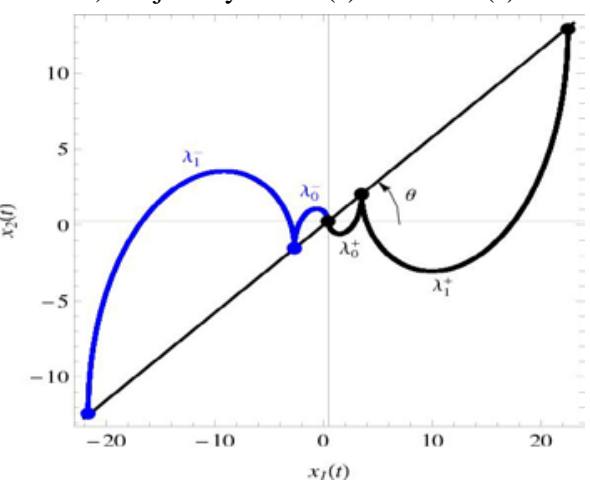

b) Optimal switching surface.

Fig. 2. Trajectory and optimal switching surface $\xi=0.5$ and $\omega_{n}=1 \mathrm{rad} / \mathrm{sec}$.

$$
u^{*}(t)= \begin{cases}u_{N} & S_{o p}\left(x_{1}, x_{2}\right)<0 \\ u_{P} & S_{o p}\left(x_{1}, x_{2}\right) \geq 0\end{cases}
$$

The switching surface of the optimal SMC is a set of ellipsoid forms which are dependent on the system parameters. These ellipsoid shapes change due to variations in the system parameters, and they finally convert into circle shapes when $\xi=0$. Fig. 3 shows a typical system trajectory that can reach the desired point from an arbitrary initial state by an optimal three times input switching with sequences.

\section{Time Optimal CONTROL FOR NEW MATRIX FORM SYSTEM}

In practice, the above relations for the minimum time optimal control are not applicable, because the optimal switching surface varies according to the load conditions. A new system matrix is introduced for modeling and practical implementation of the optimal control in damping mode conditions. New state space variables are defined as:

$$
\left(\begin{array}{c}
x_{1 \text { new }}(t) \\
x_{2 \text { new }}(t)
\end{array}\right)=\left(\begin{array}{cc}
1 / \omega_{d} & 0 \\
-\xi \omega_{n} / \omega_{d} & 1
\end{array}\right)\left(\begin{array}{l}
x_{1}(t) \\
x_{2}(t)
\end{array}\right)
$$

By using (1), (8) and the similar transformation in (21), the damped mode system can be presented by:

$$
\dot{\mathbf{x}}_{\mathbf{n}}(t)=\mathbf{A}_{\mathbf{n}} \mathbf{x}_{\mathbf{n}}(t)+\mathbf{B}_{\mathbf{n}} \mathbf{u}(t)
$$




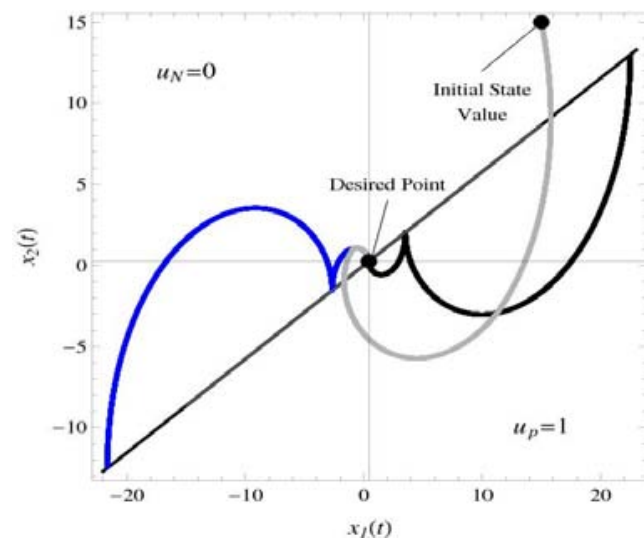

Fig. 3. Optimal trajectory with $\xi=0.5$ and $\omega_{n}=1 \frac{\mathrm{rad}}{\mathrm{sec}}$.

where:

$$
\mathbf{x}_{\mathbf{n}}(t)=\left(\begin{array}{l}
x_{\text {1new }}(t) \\
x_{\text {2new }}(t)
\end{array}\right) \quad \mathbf{A}_{\mathbf{n}}=\left(\begin{array}{cc}
0 & 1 \\
-\omega_{n}^{2} & -2 \xi \omega_{n}
\end{array}\right) \quad \mathbf{B}_{\mathbf{n}}=\left(\begin{array}{l}
0 \\
1
\end{array}\right)
$$

In this case, the Hamiltonian of the system can be given as:

$$
\begin{array}{r}
H\left(\mathbf{x}_{\mathbf{n}}(t), \mathbf{p}_{\mathbf{n}}(t), \mathbf{u}(t)\right)=1+p_{\text {1new }}(t) x_{\text {2new }}(t)+p_{\text {2new }}(t) \\
\left(u(t)-2 \xi \omega_{n} x_{\text {2new }}(t)-\omega_{n}^{2} x_{1 \text { new }}(t)\right)
\end{array}
$$

By using (11) and (24), the costate dynamical system can be obtained as:

$$
\left(\begin{array}{c}
\dot{p}_{\text {1new }}(t) \\
\dot{p}_{\text {2new }}(t)
\end{array}\right)=\left(\begin{array}{cc}
0 & \omega_{n}^{2} \\
-1 & 2 \xi \omega_{n}
\end{array}\right)\left(\begin{array}{l}
p_{\text {1new }}(t) \\
p_{\text {2new }}(t)
\end{array}\right)
$$

where time domain solution of $p_{\text {2new }}(t)$ in (25) is:

$$
p_{2 \text { new }}(t)=e^{\xi \omega_{n} t}\left(k_{3} \cos \omega_{d} t-k_{4} \sin \omega_{d} t\right)
$$

where $k_{3}$ and $k_{4}$ are two constants that can be obtained from the boundary conditions. Similar to the last section, the Hamiltonian and costate functions in (24) and (25) are the time optimal control of system (23) with the constrained input control (2). In this case, the input control is followed by the bang-bang property and the piecewise constant for $\pi / \omega_{d}$ units of time. Then, relation (19) is valid for this system by changing the system variables to (21). Fig. 4 shows a typical optimal switching surface for the new system in terms of different values of $\xi$. In this figure, some of the characteristics of the system trajectories such as the peak values of $x_{\text {2new }}(t)$ in proportion to $\xi$ and the peculiarity of point $A_{1}, A_{1}^{\prime} ? A_{2} A_{2}^{\prime} \cdots$ can be obtained by solving the response of the system with an input control of zero and the initial values $x_{\text {1new }}(0)$ and $x_{2 n e w}(0)=0$. In this condition, the system trajectory is:

$$
\begin{aligned}
& x_{\text {1new }}(t)=\frac{x_{1 \text { new }}(0)}{\sin \theta} e^{-\xi \omega_{n} t} \sin \left(\omega_{d} t+\theta\right) \\
& x_{2 \text { new }}(t)=-\frac{x_{1 \text { new }}(0)}{\sin \theta} e^{-\xi \omega_{n} t} \sin \omega_{d} t
\end{aligned}
$$

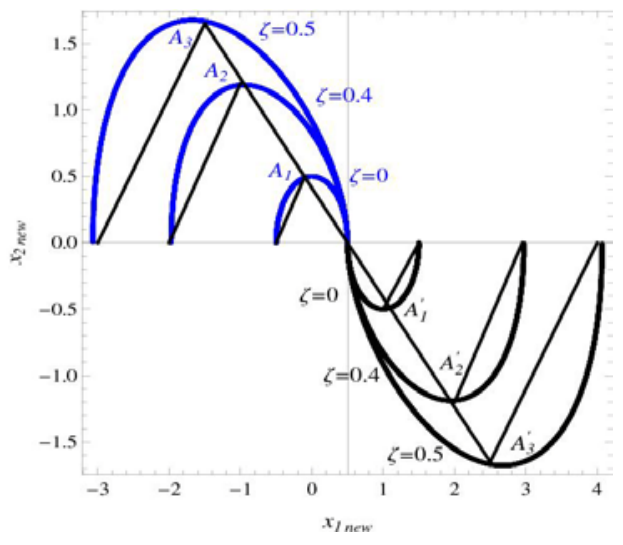

Fig. 4. Optimal switching surface with $\omega_{n}=1 \frac{\mathrm{rad}}{\mathrm{sec}}$.

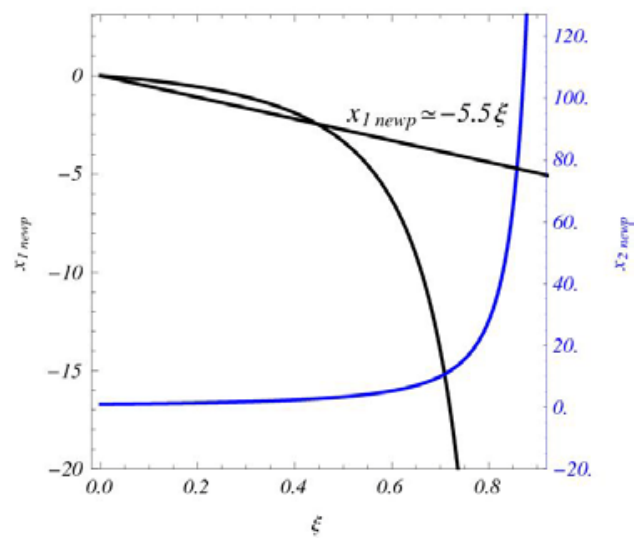

Fig. 5. Amplitude of state variables in (29) for different values of $\zeta$, at $t_{p}$.

The maximum value of $x_{2 \text { new }}(t)$ occurs at $t_{p}$ which can be obtained from $d x_{2 \text { new }}(t) / d t=0$ as:

$$
t_{p}=\frac{\theta-\pi}{\omega_{d}}
$$

Thus by using (27) and (28), the variation of $x_{1 \text { new }}(t)$ in terms of $\zeta$, which is given by $x_{1 \text { newp }}(\zeta)$, can be expressed as:

$$
x_{\text {1newp }}(\zeta)=-2 \cos \theta x_{1 \text { new }}(0) e^{\frac{\pi-\theta}{\tan \theta}}
$$

The variation of $x_{1 \text { newp }}(\zeta)$ with $x_{1 \text { new }}(0)=1$ is shown in Fig. 5 . As shown in this figure, the peak amplitudes of $x_{\text {1newp }}(\zeta)$ and $x_{2 \text { newp }}(\zeta)$ in interval $0<\xi<0.55$ can be approximated by a linear function.

$$
x_{1 \text { newp }}(\xi) \approx \pm 5.5 \xi
$$

In practice, the optimal switching curves can be approximated by a zigzag linear function. The zigzag approximated curves with different values of $\zeta$ are shown in Fig. 4. The break points of the zigzag curve are the points $A_{i}$ and $A_{i}^{\prime}, i=1,2, \cdots$ on the curves. These points vary by 


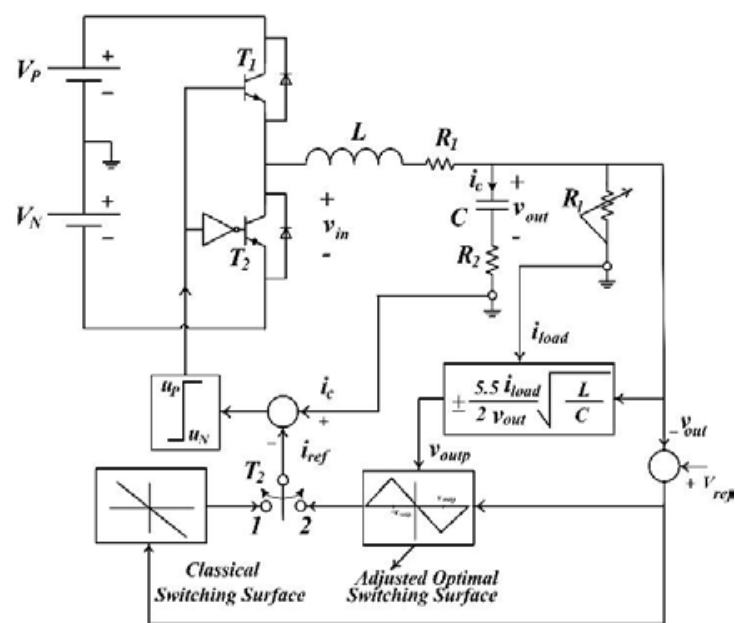

Fig. 6. Scheme of the sliding mode quadrant buck converter.

linearly changing the value of $\zeta$. However, the slope of the linear functions are independent of $\zeta$. In addition $x_{1 \text { newp }}(\zeta)$ is the break point of the zigzag curve which is approximated by (30). Thus, the zigzag switching surfaces have similar shapes but the break points are linearly dependent on $\zeta$.

\section{Optimal CONTROLler IN MUlti-QuadRANT BUCK CONVERTERS}

By real implementation, the dynamic performances of the closed-loop system in classical and optimal SMC controllers are compared and evaluated in multi-quadrant buck converters. Fig. 6 shows the scheme of a closed-loop SMC in a multi-quadrant buck converter with classical and optimal switching surfaces. Using $v_{c}(t)$ and $i_{c}(t) / C$ as the state variables of the system, the state space model of the multi-quadrant buck converter in Fig. 6 can be obtained as (22). In this case, the following is obtained:

$$
\left(\begin{array}{c}
\dot{v}_{c}(t) \\
\frac{1}{C} \dot{i}_{c}(t)
\end{array}\right)=\left(\begin{array}{cc}
0 & 1 \\
-\omega_{n}^{2} & -\xi \omega_{n}
\end{array}\right)\left(\begin{array}{c}
v_{c}(t) \\
\frac{1}{C} i_{c}(t)
\end{array}\right)+\left(\begin{array}{l}
0 \\
1
\end{array}\right) u(t)
$$

where:

$$
\begin{gathered}
\xi=\frac{K_{1}}{2 \omega_{n}}\left(R_{1}+R_{2}+\frac{R_{1} R_{2}}{R_{l}}+\frac{L}{R_{l} C}\right) \\
\omega_{n}=\sqrt{\left(\frac{1}{C}+\frac{R_{1}}{R_{l} C}\right) K_{1}} \quad K_{1}=\frac{1}{L} \frac{R_{l}}{R_{l}+R_{2}}
\end{gathered}
$$

where $C, L, R_{1}, R_{2}$ and $R_{l}$ are the capacitance, inductance, inductor resistance, capacitor leakage resistance and load resistance of the converter, respectively. In addtion, the input control of the converter is given by:

$$
u(t)=\frac{K_{1}}{C} v_{\text {in }}(t)
$$

Based on the structure of the converter, the constraint on the input control $u(t)$ can be taken by:

$$
\frac{K_{1}}{C} V_{N} \leq u(t) \leq \frac{K_{1}}{C} V_{P}
$$

In this inequality the constraints, $V_{P}$ and $V_{N}=\left\{0,-V_{p}\right\}$ are the positive and negative input source voltages of the converter, respectively. The load current $i_{\text {load }}(t)$ is an output variable in any multi quadrant buck converter which is disturbed by input and output variations. To eliminate the disturbance in the minimum time, the optimal switching surface could be obtained as in the last section. The final values of the state variables are considered as:

$$
v_{c}\left(t_{f}\right)=V_{\text {ref }} \quad i_{c}\left(t_{f}\right)=0
$$

Using (3), (31) and (35), the switching surface for the classical SMC can be obtained as:

$$
S_{s m}\left(v_{c}, i_{c}\right)=\beta_{1}\left(v_{c}-V_{r e f}\right)+\frac{\beta_{2}}{C \omega_{n}} i_{c}
$$

For $\beta_{1}=1 / R_{l}$ and $\beta_{2}=C \omega_{n}$ [5], relation (36) can be represented as:

$$
S_{s m}\left(v_{c}, i_{c}\right)=i_{c}-i_{\text {ref }}=i_{c}-\frac{1}{R_{l}}\left(V_{\text {ref }}-v_{c}\right)
$$

Moreover, the optimal switching surface $\left(s_{o p}\left(v_{c}, i_{c}\right)\right)$ can be obtained as the zigzag curve shown in Fig. 4. By substituting (32) into (30) and neglecting $R_{1}$ and $R_{2}$ comparison with $L$ / $C$, the break point of the zigzag curve can be obtained as:

$$
V_{c p}= \pm \frac{5.5}{2 R_{l}} \sqrt{\frac{L}{C}}
$$

As shown in Fig. 6, the state of $x_{1 \text { newp }}(t)=v_{c p}(t)$ is a linear function of $\xi$, (Eq. 30) in the interval of $0<\xi<0.55$. The damping ratio $\xi$ is directly proportional to $1 / R_{l}$. Then, for the given output voltage, the load current is directly proportional to $\xi$. Thus the time optimal switching surface can be adjusted to the output load which is shown in Fig. 6.

In passive resistance loads, the sign of the output voltage and the current of the converter are the same, and the break points of the zigzag switching curve are proportional to $i_{\text {out }} / v_{\text {out }}$. Thus the break points can be adjusted by using analogue divider circuits. In addition, a quadrant Operational Transconductance Amplifier (OTA) is especially suited for many low frequency, low-power four-quadrant multiplier and divider applications. An OTA is similar to conventional operational amplifiers but the output of an OTA is a current signal [18]. The output current of an OTA is proportional to the transconductance gain and the differential input voltage. The transconductance gain is adjusted by the amplifier bias current. The divider circuit in Fig. 7-a is realized by using an OTA and the input-output transfer characteristic of this circuit is recorded in Fig. 7-b. The transfer characteristic of this circuit is very similar to the zigzag switching curve in Fig. 4 and the closed-loop optimal sliding mode control system in Fig. 6. 


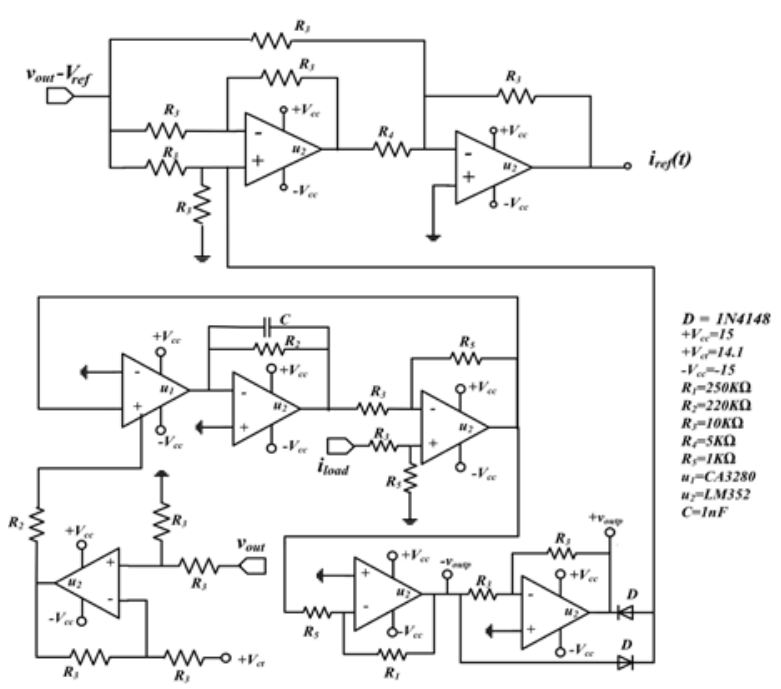

(a) Circuit.

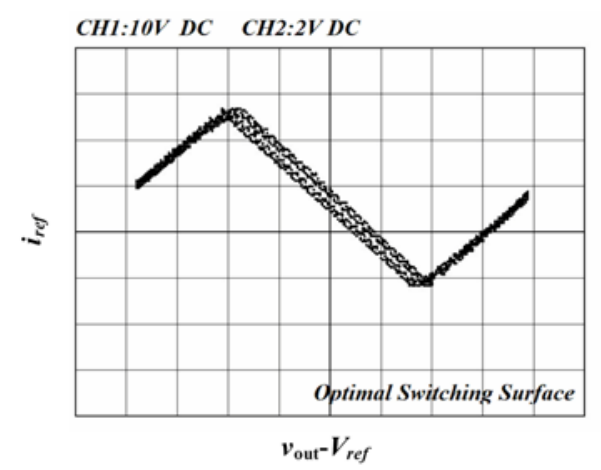

(b) Transfer characteristic.

Fig. 7. Controller for time optimal switching surface.

\section{NUMERICAL SIMULATIONS AND EXPERIMENTAL RESULTS}

Figs. 8-a and 8-b show the simulation results for the classical sliding, exact and approximated optimal SMC, when the load of converter is decreased from $R_{l}=0.3 \Omega$ to $R_{l}=5 \Omega(\xi=0.5)$. The input and reference voltages are $V_{p}=20 \mathrm{volts}, V_{N}=0$ and $V_{\text {ref }}=10 \mathrm{volts}$ and the converter parameters are presented in Table I. These results show that the approximated optimal switching surface is a proper response to damp the output disturbance in a buck converter in the minimum time. However, the exact and approximated optimal SMC reach the final value in $70 \mu$ s which is sooner than the classical SMC with a slope of 1/0.3 .

In addition, other simulation results are done for a converter with parameter similar to those in Table I in $V_{p}=10$ volts, $V_{N}=0$ and $V_{\text {ref }}=5$ volts. In this case, the output load changes from $R_{l}=2.5 \Omega$ to $R_{l}=5 \Omega(\xi=0.5)$. The output voltage, the capacitor current and the system trajectory are shown in Figs. 9-a to 9-c. These figures illustrate that the disturbance of the output voltage is damped in about $t=25 \mu \mathrm{s}$

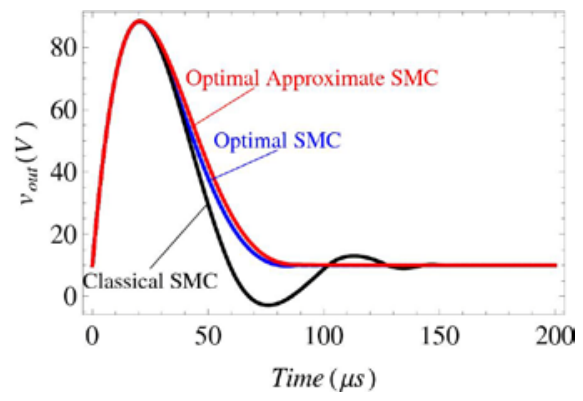

(a) Output voltage.

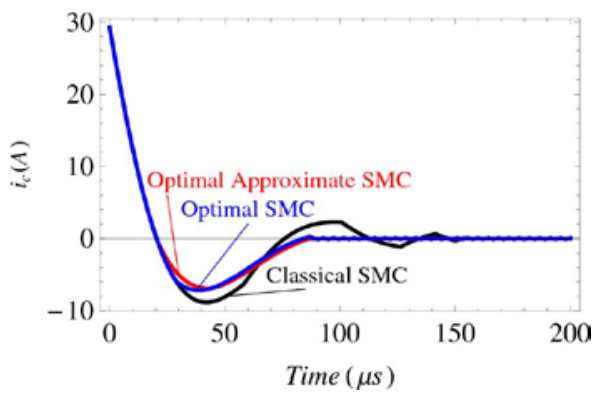

(b) Capacitor current.

Fig. 8. Classical and optimal SMC with $V_{P}=20 v$ and $V_{N}=0$ and load step decreased from 0.3 to 5 .

for the optimal sliding mode control. However, this occurs in more than $t=100 \mu \mathrm{s}$ for the classical sliding mode control with a slope of $\beta_{1}=0.2$.

The experimental results of the system are shown in Figs. $10-\mathrm{a}$ and 10-b. In these figures, Ref 1 and Ref 2 are representations of the ground levels for $\mathrm{CH} 1$ and $\mathrm{CH} 2$. Furthermore, the maximum magnitudes of the output over the voltage for the optimal and classical SMC are similar. Under step up/down load variations, the storage energy in the inductor/capacitor is transferred to the capacitor/inductor and a transient over/under voltage appears in the output voltage. Therefore, the output load connection to the output of a converter may be damaged. Thus optimal regulation of the minimum over-voltage magnitude and the transient time is an important property of closed-loop multi-quadrant buck converters. To compare the transient performances of the two closed-loop systems, a transient over-voltage index can be introduced as:

$$
j(t)=\int_{0}^{t}\left(v_{c}(t)-v_{r e f}\right)^{2} d t
$$

As shown in Figs. 11-a and 11-b, the transient over-voltage index for the optimal SMC is less than that of the classical SMC. The above-mention results for a multi-quadrant buck converter with $V_{p}=10$ volts, $V_{N}=-10$ volts are shown in Figs. 12-a and 12-b, when the converter load increases from $R_{l}=10 \Omega$ to $R_{l}=5 \Omega$.

Thus the time optimal controller can be applied to damp disturbances with minimum time and energy for multi-quadrant buck converters. 


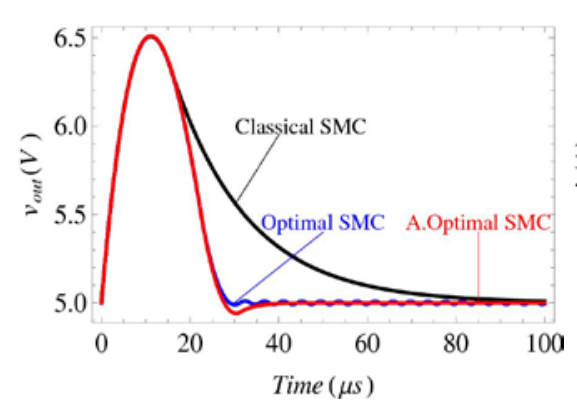

(a) Output voltage.

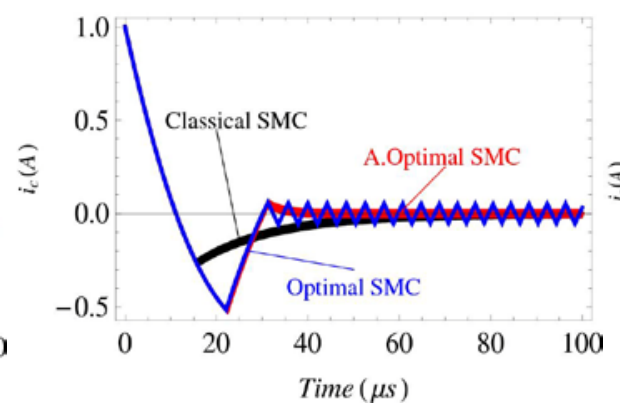

(b) Capacitor current.

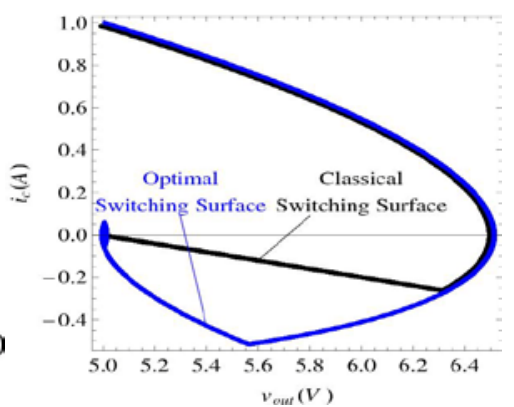

(c) System trajectory.

Fig. 9. Classical and optimal SMC with $V_{p}=10 \mathrm{v}, V_{N}=0$ and load step decreased from $2.5 \Omega$ to $3 \Omega$.

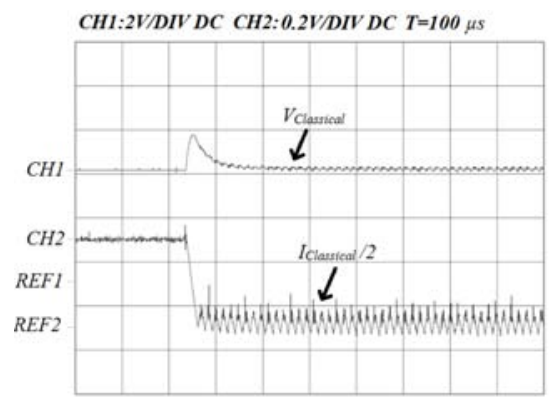

(a) Classical SMC.

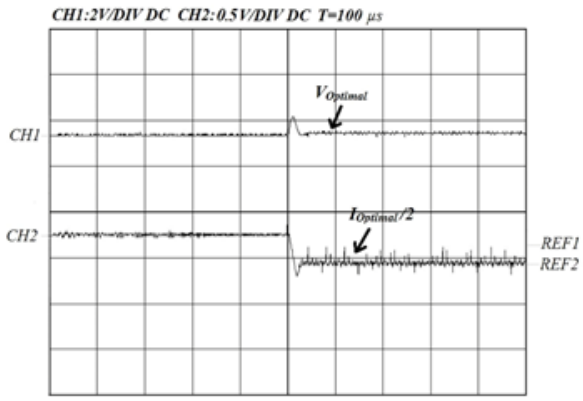

(b) Time optimal SMC.

Fig. 10. Experimental results for output voltage and capacitor current.

\section{CONCLUSIONS}

This paper presents the design of a time optimal sliding mode closed-loop controller for the class of second-order systems with stable damped harmonic oscillator systems. An adjusted time optimal controller was suggested to make the optimal switching surface correspond to every load variation. Multi-quadrant buck converters were used to verify the validity of the design procedure. By substituting the optimal switching surface for the classical one, the output voltage could be regulated in a minimum time. Moreover, the transient over-voltage index in the optimal SMC is considerably decreased.

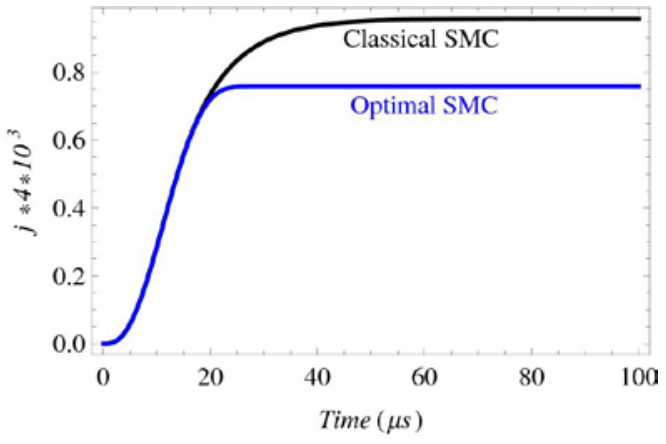

(a) Load decreased from $R_{l}=2.5 \Omega$ to $R_{l}=5 \Omega$.

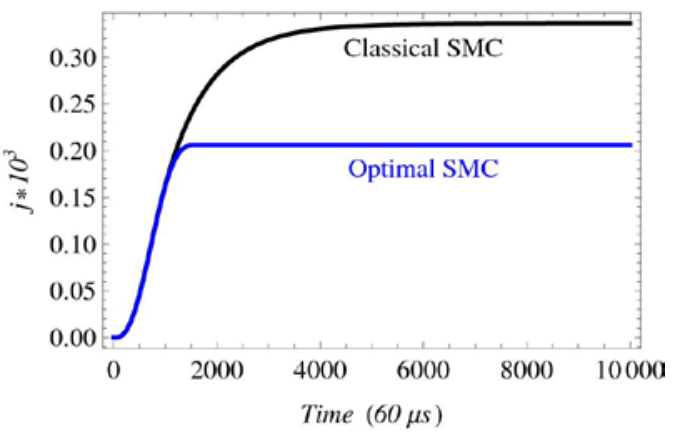

(b) Load increased from $R_{l}=10 \Omega$ to $R_{l}=5 \Omega$.

Fig. 11. Transient over voltage energy index for classical and time optimal SMC.

TABLE I

CONVERTER PARAMETERS

\begin{tabular}{|c|c|c|}
\hline Description & Parameter & Value \\
\hline Capacitance & $C$ & $3 \mu F$ \\
Inductance & $L$ & $96 \mu h$ \\
Load resistance & $R_{l}$ & $\{0.3,2.5,5,10\} \Omega$ \\
Inductor resistance & $R_{1}$ & $0.2 \Omega$ \\
Capacitor & $R_{2}$ & $10^{-8} \Omega$ \\
resistance & & \\
\hline
\end{tabular}




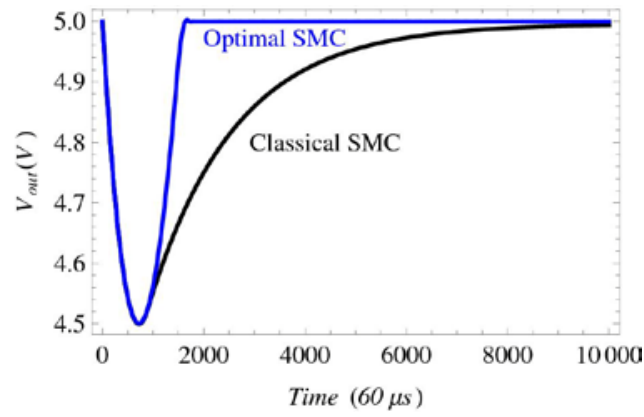

(a) Output voltage.

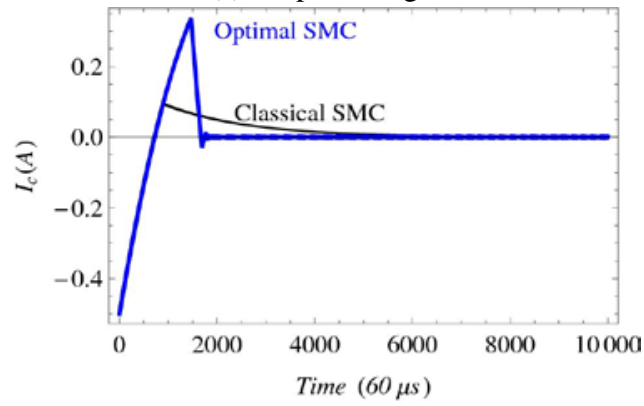

(b) Capacitor current.

Fig. 12. Classical and optimal SMC with $V_{p}=10$ volts and $V_{N}=-10$ volts and load step increased from $R_{l}=10 \Omega$ to $R_{l}=5 \Omega$

\section{REFERENCES}

[1] V. Utkin, J. Guldner, and J. X. Shi, Sliding Mode Control In Electromechanical Systems, Second Edition, CRC Press, Taylor and Francis Group, 2009.

[2] I. Zinober, Variable Structure and Lyapunov Control, Lecture Notes in Control and Information Sciences, Springer-Verlag, Berlin, 1994.

[3] L. Skvarenina, The Power Electronics Handbook, CRC Press, 2002.

[4] I. Eker, "Sliding mode control with PID sliding surface and experimental application to an electromechanical plant," ISA Transactions, Vol. 45, No. 1, pp. 109-118, Jan. 2006.

[5] S.-C. Tan, Y. M. Lai, M. K. H. Cheung, and C. K. Tse, “On the practical design of a sliding mode voltage controlled buck converter,” IEEE Trans. Power Electron., Vol. 20, No. 2, pp. 425-437, Mar. 2005.

[6] Y. He, W. Xu and Y. Cheng, "A novel scheme for sliding-mode control of dc-dc converters with a constant frequency based on the averaging model," Journal of Power Electronics, Vol. 10, No. 1, pp. 1-8, Jan. 2010.

[7] S.-C. Tan, Y. M. Lai, and C. K. Tse, "Indirect sliding mode control of power converters via double integral sliding surface,” IEEE Trans. Power Electron., Vol. 23, No. 2, pp. 600-611, Mar. 2008.

[8] E. Kirk, Optimal Control Theory: An Introduction, Dover Publications Inc., Mineola, New York, 2004.

[9] T. Chatchanayuenyong and M. Parnichkun, "Neural network based-time optimal sliding mode control for an autonomous underwater robot," Mechatronics, Vol. 16, pp. 471-478, Feb. 2006.

[10] M. J. Jafarian and J. Nazarzadeh, "Time-optimal sliding-mode control for multi-quadrant buck converters," Power Electronics, IET, Vol. 4, No. 1, pp. 143-146, Jan.
2011.

[11] S.-C. Tan, Y. M. Lai, C. K. Tse, and M. K. H. Cheung, "Adaptive feedforward and feedback control schemes for sliding mode controlled power converters," IEEE Trans. Power Electron., Vol. 21, No. 1, pp. 182-192, Jan. 2006.

[12] P. Mattavelli, L. Rossetto, G. Spiazzi, and P. Tenti, "General-purpose sliding-mode controller for dc/dc converter applications,” In Proc. IEEE PESC, pp. 609-615, 1993.

[13] E. M. Navarro-LÂ'opez, D. Cortes, and C. Casto, "Design of practical sliding mode controllers with constant switching frequency for power converters," Electric Power Systems Research, Vol. 79, pp. 796-802, Dec. 2009.

[14] S. Kapat and P. Krein, "Improved time optimal control of a buck converter based on capacitor current," IEEE Trans. Power Electron., Vol. 27, No. 23, pp. 1444-1454, Mar. 2012.

[15] M. Ordonez, T. Iqbal, and E. Quaicoe, "Selection of a curved switching surface for buck converters," IEEE Trans. Power Electron., Vol. 21, No. 4, pp. 1148-1153, Jul. 2006.

[16] S. Leung and S. Chung, "Derivation of a second-order switching surface in the boundary control of buck converters,” IEEE Power Electronics Letter, Vol. 2, No. 2, pp. 63-67, Jun. 2004.

[17] M. Athens and L. Falb, Optimal Control: An Introduction To The Theory And Its Applications, McGraw-Hill, New York, 1966.

[18] E. Sanchez-Sinencio, J. Ramirez-Angulo, B. Linares-Barranco, and A. Rodriguez-Vazquez, "Operational transconductance amplifier-based nonlinear function syntheses,” IEEE J. Solid-State Circuits, Vol. 24, No. 6, pp. 1576-1586, Dec. 1989.

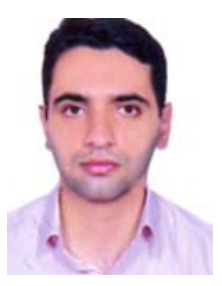

Mohammad Javad Jafarian was born in Babol, Iran, in 1983. He received his B.S. in Electrical Engineering from Mazandaran University, Babol, Iran, in 2006 and his M.S. in Electrical Engineering from the University of Shahed, Iran, in 2009. His current research interests include optimal control theory and applications, power electronics and applied

mathematics.

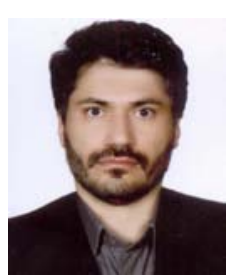

Jalal Nazarzadeh was born in Tehran, Iran, in August 1964. He received his B.S., M.S. and Ph.D. from the Amir-Kabir University of Technology, Tehran, Iran, in 1990, 1992 and 1998, respectively. In February 1998, he joined the Faculty of Engineering, Department of Electrical Engineering, Shahed University, Tehran, Iran. His current research interests include the modeling, analysis and control of electrical machines and drives, spectral theories in non-linear optimal control systems, and non-linear phenomena in electrical machines and power electronic systems. He has published 30 journal and 39 conference papers. He is the Vice President of Academic Affairs and Graduate Studies at Shahed University. 